\title{
SHOCK WAVES OF THE LARGE-SCALE STRUCTURE FORMATION IN THE UNIVERSE
}

\author{
T.A. ENSSLIN AND P.L. BIERMANN \\ Max-Planck-Institut für Radioastronomie Bonn, Germany \\ AND \\ U. KLEIN AND S. KOHLE \\ Radioastronomisches Institut der Universität Bonn, Germany
}

\begin{abstract}
Simulations of structure formation in the Universe predict accretion shock waves at the boundaries of the large-scale structures as sheets, filaments, and clusters of galaxies. If magnetic fields are present at these shocks, particle acceleration should take place, and could contribute to the observed cosmic rays of high energies. When the radio plasma of an old invisible radio lobe is dragged into such a shock wave, the relativistic electron population will be reaccelerated and the plasma becomes radio-luminous again. Such tracers of the accretion shock waves are observed at the boundaries of some clusters of galaxies: the so-called cluster radio relics, which are large regions of diffuse radio emission, without any parent galaxy nearby. The observed properties of the cluster radio relics are naturally explained by accretion shock waves. Radio relics therefore give the first evidence for the existence of accretion shocks of the large-scale structure formation and they allow investigations of the shock properties.
\end{abstract}

\section{Shock Waves of the Large-Scale Structure Formation}

The large-scale structure of the Universe, seen in the structured galaxy distribution, is still forming. Matter is flowing out of the cosmic voids onto sheets and filaments. Within the filaments the matter flows to the density cusps frequently located at the intersection points of filaments: the clusters of galaxies. Whenever the flow passes from one structure into another, its velocity suddenly changes and several Mpc sized shock waves occur. At these shock waves the kinetic energy of the gravitationally accelerated gas is 
dissipated, mainly thermalized to temperatures of a few $0.1 \mathrm{keV}$ in filaments and several $\mathrm{keV}$ in clusters of galaxies. The shock velocity at filaments is expected to be of the order of several $100 \mathrm{~km} \mathrm{~s}^{-1}$, and the accretion shocks at clusters $1000-2000 \mathrm{~km} \mathrm{~s}^{-1}$. From simulation of structure formation rough values of the cluster accretion shock radius and velocity can be given in terms of the cluster temperature as an indicator of the gravitational potential (Kang et al. 1997):

$$
\begin{aligned}
r_{\mathrm{s}} & =4.24 h_{50}^{-1} \mathrm{Mpc}\left(k T_{\text {obs }} / 6.06 \mathrm{keV}\right)^{1 / 2}(1+z)^{-3 / 2} \\
V_{\mathrm{s}, \text { predicted }} & =1750 \mathrm{~km} \mathrm{~s}^{-1}\left(k T_{\text {obs }} / 6.06 \mathrm{keV}\right)^{1 / 2}
\end{aligned}
$$

The dissipated accretion power per shock surface is:

$$
Q_{\text {flow }} \approx \frac{1}{2} n_{\mathrm{e}} m_{\mathrm{p}} V_{\mathrm{s}}^{3} \approx 4 \cdot 10^{44} \frac{\mathrm{erg} \mathrm{s}^{-1}}{\mathrm{Mpc}^{2}} \frac{n_{\mathrm{e}}}{10^{-5} \mathrm{~cm}^{-3}}\left(\frac{k T_{\mathrm{obs}}}{6.06 \mathrm{keV}}\right)^{3 / 2} .
$$

\section{Particle Acceleration at Cluster Accretion Shocks}

If magnetic fields are present at the location of the accretion shocks, charged particles are accelerated. The acceleration of protons is mainly limited by photo-meson production to $<10^{20} \mathrm{eV}$, but could contribute to the UHECR spectrum below this energy (Kang et al. 1997). Relativistic electrons lose their energy by IC scattering of MWB photons and synchrotron losses. Especially the synchrotron radio emission should be a sensitive tracer of shock waves. The best detectability is given if a strongly magnetized plasma is present with a preaccelerated population of electrons. This is the case whenever an old remnant radio lobe of a former radio galaxy is dragged into such a shock. Since the Universe was sufficiently polluted by radio plasma at the epoch of quasar activity (Enßlin et al. 1997, 1998b), such shocks should be traceable today. We argue that this is the case for cluster radio relics.

\section{Cluster Radio Relics}

- Peripherally located sources of irregular extended radio emission.

- Steep radio spectra $\alpha=1.0-1.5$.

- Typical radio luminosities of $10^{41 \ldots 42} \mathrm{erg} \mathrm{s}^{-1}$.

- Radio relics are believed to be the remnants of radio lobes of radio galaxies, where the former active galaxy has become inactive or has moved away.

- But cluster radio relics have frequently no nearby possible parent galaxy within an electron cooling-time travel distance.

- Further: The radio spectra of cluster radio relics do usually not show an apparent cutoff, as the spectra of old remnants of radio galaxies do. 


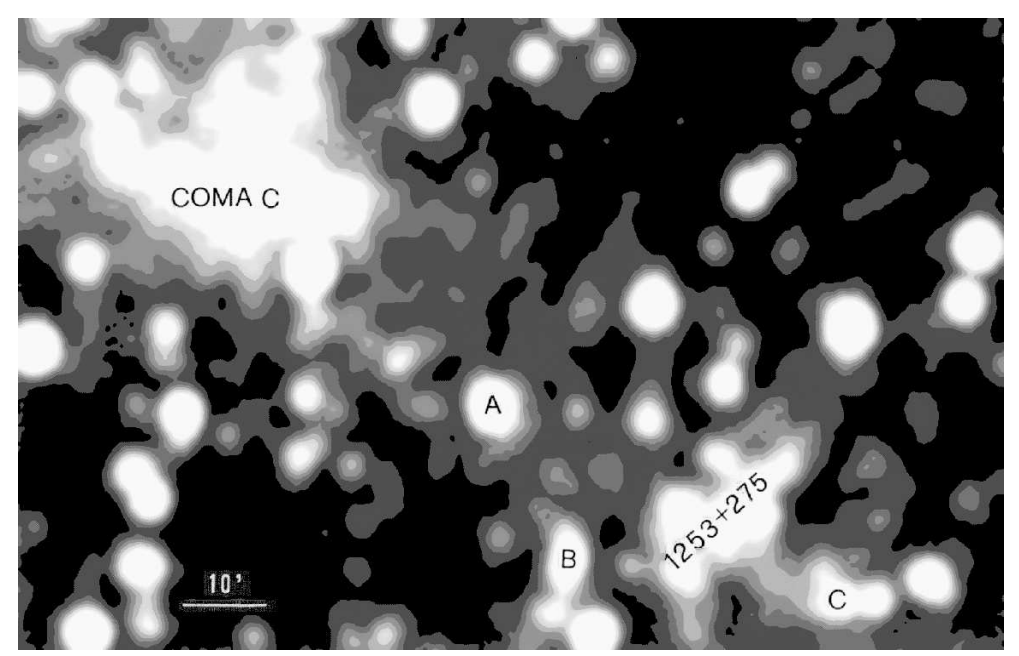

Figure 1. Westerbork Synthesis Radio Telescope map at $327 \mathrm{MHz}$ of the Coma cluster from Giovannini et al. (1991). The central halo source Coma C and the cluster relic $1253+275$ are identified. Capital letters indicate some extended Coma cluster galaxies, as following: $\mathrm{A}=\mathrm{NGC} 4839, \mathrm{~B}=\mathrm{NGC} 4827, \mathrm{C}=\mathrm{NGC} 4789.10^{\prime} \approx 400 \mathrm{kpc} h_{50}^{-1}$.

- Thus the electron population is (re)accelerated there.

- Nine known examples: 0038-096 in A85, 0917+75 in A786, 1140+203 in A1367, 1253+275 in Coma, 1712+64 in A2255, 1706+78 in A2256, 2006-56 and 2010-57 in A3667, 1401-33 in S753

\section{A Simple Model}

The orientation of the magnetic fields of the infalling plasma is assumed to be distributed randomly. The plasma is compressed at the shock by the compression ratio $R$. This determines the spectral index of the electron population. The high energy cutoff of the downstream electron population is a function of the distance to the shock plane. High resolution radio observations should therefore reveal a flatter (steeper) spectrum at the outer (inner) edge of the relic, which is closer (more distant) to the accretion shock. This is indeed observed for the relics in Coma and A3667. The overall radio spectrum is a superposition of the spectra at different distances and is composed by a flat low frequency spectrum, then a spectral index steeper by 0.5 due to the superposition of different cutoffs, and a final cutoff. The observed spectral indices of relics belong to the steeper region, expect in the case of the relic in A2256, which is not due to a peripheral accretion shock but due to a massive cluster merger. The shock compression ratio 


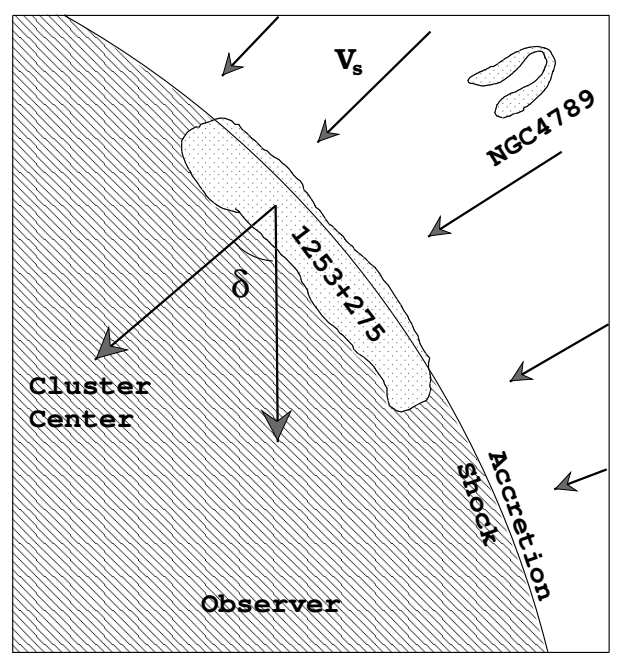

Figure 2. Sketch of the assumed geometry in the Coma cluster. The accretion flow is indicated by arrows. The shaded region is the intra-cluster medium. The dotted area represents regions of radio plasma. The line-of-sight and the line connecting the relic and the cluster center are lying within the plane of the figure. NGC4789 is placed upstream above the radio relic as a source of relativistic electrons. Its line-of-sight velocity component points away from the observer.

can be calculated ${ }^{1}$ from the observed radio spectrum:

$$
R=(\alpha+1) /(\alpha-0.5),
$$

The ratio of break- to cutoff-frequency allows (if observed) a rough estimate of the electron diffusion coefficient (Enßlin et al. 1998a):

$$
\kappa_{2} \approx V_{\mathrm{s}} D R^{-1} \sqrt{\nu_{\mathrm{break}} / \nu_{\mathrm{cut}}}<3 \cdot 10^{30} \mathrm{~cm}^{2} \mathrm{~s}^{-1} .
$$

The thickness $D \approx 0.1 \mathrm{Mpc}$, and the other parameters are taken for the relic $1253+275$ in Coma. The thickness divided by the post shock velocity $V_{\mathrm{s}} / R$ gives the age of the relic after it passed the shock. It should be similar to the cooling time of electrons visible at the spectral break. Both ages agree roughly for all known relics to be of the order of $\approx 10^{8} \mathrm{yr}$. The shock efficiency which is necessary in order to power the radio emission is $0.1 \ldots 5 \%$.

\footnotetext{
${ }^{1}$ Since particle acceleration is most efficient for oblique shocks (Jokipii 1987), patches with magnetic fields oriented parallel to the shock plane will dominate the acceleration. The spatial diffusion coefficient, which determines the efficiency of the acceleration process, is only poorly understood in such complicated circumstances. Thus, we assume a momentum independent coefficient, because of the success of this simplification in the similarly complicated case of SNR CR-acceleration (Biermann 1993).
} 

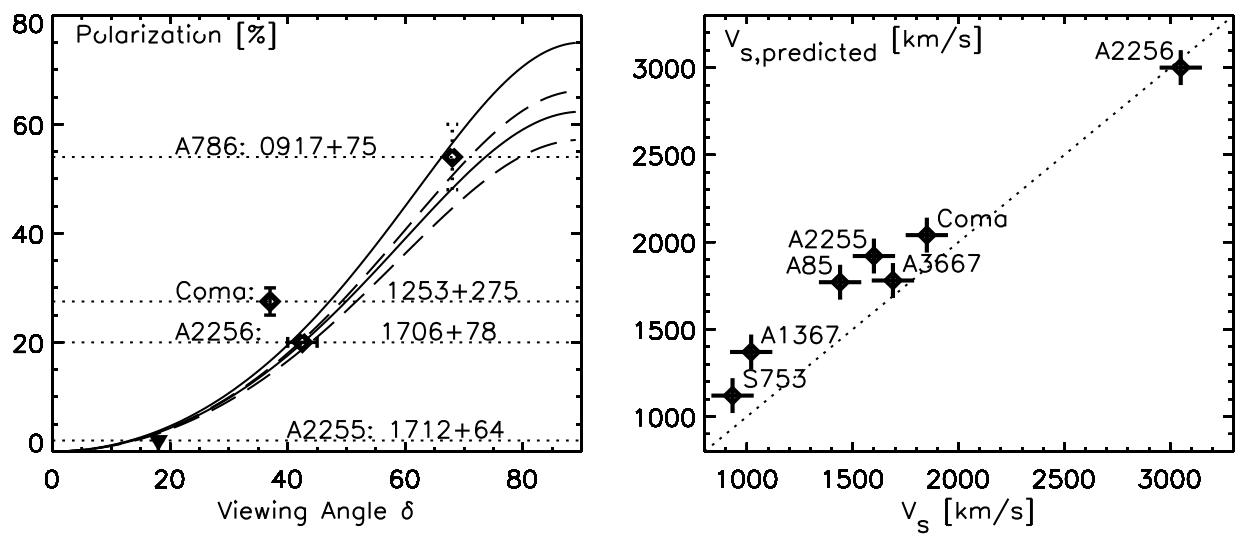

Figure 3. Polarization of synchrotron emission of shocked magnetic fields as a function of the viewing angle $\delta$. The curves are theoretical predictions for different radio spectral indices and different magnetic field strength (Enßlin et al. 1998a). Observed polarizations of radio relics are plotted above the viewing angle predicted from the accretion shock theory $(1253+257,1712+64)$, simulations of a cluster merger $(1706+78$, Roettiger et al. (1995)), or - if no data are available - at their best-fit position $(0917+75)$. The large observed projected radius of $0917+75$ indicates a large viewing angle, consistent with the best-fit position. Uncertainties in the angles are large.

Figure 4. A comparison of the shock velocity predicted from simulations of structure formation $V_{\mathrm{s} \text {,predicted }}$ (Eq. 2), and $V_{\mathrm{s}}$ estimated using the derived compression ratio from the relic spectral index. The good agreement demonstrates self-consistency of our simple model. Error bars are large. In the case of A2256 the predicted shock velocity is derived by Roettiger et al. (1995) by fitting cluster merger scenarios to the observed X-ray profiles.

\section{Radio Polarization}

The magnetic fields of the old radio lobe get compressed in the shock and therefore aligned with the shock plane. If the shock is observed inclined by a viewing angle $\delta$ the projected fields are perpendicular to the projected shock plane. For relics with observed radio polarization (Coma, A2256, A786) this is true. If the magnetic fields are not dynamical important, the radio polarization of fields, which were originally randomly oriented, can be estimated (Enßlin et al. 1998a). Since an independent very rough estimate of the viewing angle $\delta$ is available from Eq. 1 and the projected observed radius, the expected correlation between polarization and expected viewing angle can be checked (Fig. 3).

\section{Probing Large-Scale Flows}

Cluster radio relics allow to measure properties of the large-scale flows. An extrapolation of the cluster gas density by the usual $\beta$-profile divided by the compression ration gives an estimate of the density of the infalling gas: $n_{\mathrm{e}} \approx$ 
$0.5 \ldots 1.0 \cdot 10^{-5} \mathrm{~cm}^{-3}$. The temperature jump of the shock is also given by the compression ratio. Assuming that the temperature inside the shock is half of the central cluster temperature, which is a typical observed decrease, one gets for the temperature of the infalling gas $0.5 \ldots 1 \mathrm{keV}$. Also the predicted shock velocities (Eq. 2) can be checked: Using the observed parameters of the shock the velocity $V_{\mathrm{s}}$ of the infalling matter can be estimated. This is consistent with the prediced one $\left(V_{\mathrm{s} \text {,predicted }}\right)$ from cosmological structure formation (Fig. 4).

\section{Conclusions}

- Giant shock waves of the cosmological large-scale motion of the ongoing structure formation are places of particle acceleration.

- The conditions for particle acceleration are ideal if an old radio lobe is dragged into such a shock wave.

- First observational evidence for these shock waves is presented: Cluster radio relics trace accretion shock fronts where the gas of large-scale filaments flows into clusters of galaxies

- Observed properties of cluster radio relics can be explained if they are understood as remnant radio plasma within cluster accretion shocks.

- Properties of the large-scale flows can be measured.

- The long lasting outstanding problem of the energy supply of cluster radio relics is solved by the accretion shock theory.

\section{References}

Biermann P.L., 1993, A\&A 271, 649

Jokipii J.R., 1987, ApJ 313, 842

Enßlin T.A., Biermann P.L., Kronberg P.P., Wu X.-P., 1997, ApJ 477, 560

Enßlin T.A., Biermann P.L., Klein U., Kohle S., 1998a, A\&A 332, 395

Enßlin T.A., Wang Y., Nath B.B., Biermann P.L., 1998b, A\&A 333, L47

Giovannini G., Feretti L., Stanghellini C., 1991, A\&A 252, 528

Kang H., Rachen J.P., Biermann P.L., 1997, MNRAS 286, 257

Roettiger K., Burns J.O., Pinkney J., 1995, ApJ 453, 634 